\title{
Concentration du fluage avec le temps
}

\section{Prof. George} TER-STEPANIAN

(Dr. Sc. Eng.)

Membre Correspondant de l'Académie Sciences d'Arménie
Le sol dans le domaine du fluage montre différents comportements rhéologiques sous des niveaux moyens ou hauts de contrainte de cisaillement. Pour des contraintes de cisaillement moyennes après quelques dizaines de jours de fluage à vitesse décroissante, la vitesse de fluage croît pendant quelques centaines de jours, puis elle décélère à nouveau jusqu'à ce qu'il se produise une stabilisation. Pour des contraintes de cisaillement hautes, le fluage commence par décélérer pendant quelques dizaines de jours puis il accélère sans cesse jusqu'à la rupture complète, ce qui parfois peut prendre plusieurs années. Ainsi la frontière entre les zones de contraintes moyennes et hautes divise le sol en deux domaines qui se développent dans différentes directions. Il en résulte qu'une concentration du fluage avec le temps se produit, les grandes zones de fluage se transformant en bandes étroites, qui se rattachent aux surfaces de glissement potentielles et la rupture peut se produire après une longue durée.

\section{Creep concentration with time}

The soil in the creep zone shows different rheological behavior at medium and high shear stress levels. At medium shear stresses, after several tens of days of decelerating creep, the creep rate increases during several hundreds of days, then the creep rate decelerates again and the soil comes to stabilization. At high shear stresses the creep first decelerates during several tens of days, then it accelerates incessantly up to total rupture, which may need sometimes years. Therefore the boundary between the medium and high shear stress zones divides the soil body into two parts developing into different directions. As a result the creep concentration with time occurs, the wide creep zones transform into narrow strips, attached to the potential surfaces of sliding and the rupture may take place after long time. 




Temps $t$

FG.1 Interprétation traditionnelle des courbes de fluage des sols (d'après Schmid, 1962).

Traditional interpretation of soil creep curves (after Schmid, 1962).

\section{1}

\section{Considérations théoriques}

\section{1}

\section{La conception traditionnelle des sols considérés comme des corps quasi solides}

D’après les principes de la mécanique des sols, le sol est considéré comme un corps quasi solide oủ les contraintes et les déformations sont des fonctions différentiables et continues des coordonnées de l'espace et du temps, ce qui signifie qu'elles obéissent aux conditions de continuité ou de déformations simultanées (Goldstein, 1979). Les déformations du sol au cours des variations de l'état de contrainte ou du temps sont des processus déterministes non-linéaires qui s'expriment par des courbes régulières. La déformation de tels corps se produit à un seul niveau.

Ces hypothèses rendent possible d'appliquer à la mécanique des sols les méthodes mathématiques bien développées utilisées en mécanique des milieux continus: théories de l'élasticité, de la plasticité, du fluage. Les résultats d'une telle approche sont les solutions déterministes bien connues aù les relations entre les contraintes, les déformations et le temps sont exprimées par des fonctions exponentielles ou puissances. Une conséquence de cette approche est que les acquisitions dans le domaine de la science des sols qui ont trait à la texture et à la structure, obtenues par les méthodes modernes d'analyse comme la microscopie électronique, l'analyse aux rayons X, l'étude des liaisons magnétiques, l'analyse thermique des minéraux et autres, restent sans relation avec les conceptions considérées en mécanique des sols.

Le résultat de l'interprétation du comportement du sol comme celui d'un corps quasi solide est la présentation traditionnelle bien connue des phénomènes de fluage lorsque les contraintes dépassent le seuil de fluage, composée de trois parties : le fluage primaire (à vitesse de déformation décroissante), le fluage secon- daire (ou à vitesse constante), le fluage tertiaire (à vitesse croissante).

On suppose que le fluage secondaire peut se transformer en fluage décéléré ou en fluage accéléré. Si le fluage s'accélère, cela se terminera tôt ou tard par une rupture. D'après le point de vue traditionnel « La rupture peut être définie comme le point sur la courbe de fluage à partir duquel la vitesse de déformation commence à croître, c'est-à-dire où l'accélération de la déformation devient positive $(\gamma>0)$ » (Schmid, 1962). Ces points sont marqués par des cercles noirs sur la fig. 1.

Une très importante conclusion est issue de cet énoncé ; il nous oblige à limiter les contraintes de cisaillement admissible au point où l'on n'observe pas d'accélération de la déformation car il existerait alors le danger que si la vitesse de fluage se mettait à croître le sol se romprait inévitablement en fonction du temps.

Deux suppositions erronées sont faites avec une telle conclusion :

(1) La durée des essais normaux de fluage est suffisante pour être certain que le fluage secondaire se transforme en un fluage accéléré conduisant à la rupture ou au contraire en un fluage avec stabilisation. Ainsi, les résultats des essais à court terme sont surestimés puisque la transition de l'accélération ne peut pas être obtenue par des essais de courte durée.

(2) La transition vers l'accélération signifie que le processus de fluage va se terminer par la rupture; c'est faux car pour des contraintes de cisaillement moyennes la phase d'accélération est suivie par une phase de stabilisation qui survient plusieurs centaines de jours après l'application des contraintes de cisaillement et ainsi l'utilisation du critère d'accélération rend sousestimés les résultats des essais de fluage. On vérifiera plus loin que cet énoncé est correct.

Ces deux sources d'erreur agissant en sens contraire se compensent partiellement l'une l'autre et la réponse peut être acceptable. Mais c'est une question de chance. Pour des combinaisons défavorables de ces erreurs, les résultats peuvent s'écarter matériellement de la réalité et la force portante du sol peut aussi bien être sous-estimée - ce qui conduit à des décisions 




Dispersion des points expérimentaux de la courbe de fluage qui ne peut être expliquée par des erreurs de mesure.

Scattering of experimental points of the creep curve that cannot be explained by measurement errors.

qui ne sont pas économiques - ou surestimée - ce qui conduits à des projets qui ne sont pas en sécurité ou même à haut risque.

Le modèle largement répandu de Bingham pour les corps rhéologiques est basé sur la relation linéaire trouvée empiriquement entre la contrainte de cisaillement et la vitesse du fluage secondaire. En général, chaque essai de fluage en cisaillement commence par des vitesses de déformation qui diminuent en fonction du temps et se termine presque par une ligne droite (voir par exemple la fig. 10). Mais cela n'a rien à voir avec le fluage secondaire. La même partie de la courbe de fluage, qui ressemble à une ligne droite dans une échelle de temps particulière apparait effectivement courbe dans une échelle de temps plus petite. Bishop (1966) a montré l'absence de fluage secondaire d'où il suit que le modèle de Bingham n'est pas utilisable pour les sols. Cependant malgré cela ce modèle défectueux continue à être utilisé.

On ne doit pas oublier que Bingham a étudié le pouvoir couvrant des couleurs à l'huile et ne s'occupait pas des sols. L'emploi d'un modèle inadéquat pour le sol a un résultat matériel négatif c'est-à-dire que très peu d'information peuvent être obtenues par les essais de fluage. Dans l'état actuel, la recherche d'amélioration de la précision disponible des mesures n'a pas grand sens ; la précision disponible des mesures n'est même pas utilisée complètement pour obtenir des informations relatives au comportement rhéologique des sols. C'est pourquoi les résultats des essais de fluage sont représentés généralement à petite échelle.

Les résultats d'un essai de fluage drainé au cours du cisaillement, pendant les cinquante-deux premiers jours sont donnés sur la figure 2 comme une illustration de l'état du problème. Il semble que la dispersion des points du graphique montre que cela provient de mesures très grossières visibles à grande échelle. Cependant les erreurs de mesures étaient la moitié du rayon des cercles représentatifs, et la dispersion des points n'avait rien à voir avec l'imprécision des mesures. Cette affirmation sera vérifiée plus loin. Il en résulte que les progrès dans l'analyse du fluage du sol ne sont pas tant un problème technologique qu'un problème conceptuel.
Ce problème peut être formulé par les questions suivantes:

(1) Le fluage est-il un phénomène déterministe ?

(2) Quelle est la nature du fluage secondaire décrite par quelques auteurs?

(3) La transition vers le fluage accéléré signifie-t-elle ou non que la résistance limite au cisaillement a été atteinte et que le sol va inévitablement se rompre?

(4) Qu'est-ce que la résistance limite des sols?

(5) Quel est le comportement des sols lorsqu'une contrainte de cisaillement est appliquée pendant une très longue période?

\section{2}

\section{Quatre niveaux de déformations et quatre phases de fluage}

Les sols sont des systèmes dispersés complexes dans lesquels la résistance des liaisons entre les particules est considérablement moindre que celle des particules elles-mêmes.

La déformation des sols sous l'action de petites contraintes, comme celles qui s'exercent sous les fondations ou lors de la construction des barrages consiste essentiellement dans le changement de forme et de dimension des vides du sol et dans un moindre degré du changement de forme des particules elles-mêmes. Le changement de volume des particules est très petit et peut être négligé. Il en résulte que l'analyse de la déformation du sol mène à l'analyse des structures du sol.

La structure du sol, au sens rhéologique est déterminée par l'arrangement mutuel des particules dans l'espace, par la position des contacts entre les particules et par la grandeur et la direction des forces élémentaires agissant aux points de contact, assurant ainsi l'existence de liaisons stables. Les phénomènes de déformation dans un tel système passent par quatre niveaux: moléculaire, des particules, structurel et des agrégats. La déformation des sols est un exemple d'auto-organisation de la matière. 


\section{9}

\section{Le niveau moléculaire de la déformation du sol}

La déformation au niveau moléculaire consiste en un déplacement mutuel des unités d'écoulements lorsque les barrières d'énergie sont surmontées. Ce phénomène déterministe se produit avec une vitesse constante $\dot{\gamma}$ décrites par l'équation bien connue d'Eyring-Mitchell (Mitchell 1964, 1993) :

$$
\dot{\gamma}=x \frac{k T}{h} \exp \left(\frac{-E}{R_{0} T}\right) \exp \left(\frac{\lambda f_{0}}{2 k T}\right)
$$

où $\mathrm{X}$ est un coefficient de proportionnalité ; k est la constante de Boltzman $\mathrm{k}=1,38.10^{-23} \mathrm{~J} \cdot{ }^{\circ} \mathrm{K}^{-1}$; h est la constante de Planck $h=6,624.10^{-34} \mathrm{~J} . \mathrm{s}^{-1}$; T est la température absolue, ${ }^{\circ} \mathrm{K} ; \mathrm{R}_{0}$ est la constante universelle des gaz parfaits, $R_{0}=8,3144 \mathrm{~J} .{ }^{\circ} \mathrm{K}^{-1} \mathrm{~mol}^{-1} ; \lambda$ est la distance qui sépare deux positions d'équilibre successives ; $E$ est l'énergie libre d'activation; $f_{0}$ est la force moyenne agissant sur chaque unité d'écoulement.

La valeur de $f_{0}$ dans l'équation (1) n'est pas une caractéristique de la contrainte tangentielle appliquée ; c'est une quantité constante pour une structure de sol donnée.

La vitesse de fluage au niveau moléculaire pour chaque structure de sol est constante.

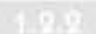

\section{Le niveau des particules de la déformation du sol}

La déformation au niveau des particules consiste en un déplacement mutuel des particules reliées à l'initiation et la rupture des liaisons entre elles, ce qui se produit sans changement appréciable de l'arrangement mutuel des particules. La part principale de ce phénomène répond à des facteurs qui sont reliés à la dispersion des sols, à la déformabilité, à la durée de vie des liaisons faibles entre les particules et au niveau de la contrainte de cisaillement.

Le rôle de ces facteurs est exprimé par différentes valeurs du coefficient X dans l'équation (1). Il a été montré antérieurement (Ter-Stepanian, 1975) que le coefficient $\mathrm{X}$ est directement proportionnel à la déformabilité F/R et au surplus du niveau de contrainte de cisaillement $\tau$ au-dessus de la valeur limite de fluage $\tau_{\text {p }}$. Il dépend aussi de la valeur moyenne de la durée de vie $\mathrm{L}$ des liaisons aux contacts entre les particules.

La nature et le rôle de ces deux premiers facteurs sont clairs. La déformabilité du sol F/R est le rapport entre la somme vectorielle des composantes tangentielles $f$ des forces élémentaires agissant entre les particules aux points de contacts sur la surface potentielle de rupture et celle des composantes normales $\mathrm{r}$ agissant sur la même surface, $\mathrm{F} / \mathrm{R}=\Sigma \mathrm{f} / \mathrm{\Sigma}$. . Les forces tangentielles rendent la structure du sol plus déformable, tandis que les forces normales la rendent moins déformable. Leur rapport est une caractéristique de la déformabilité du sol. La dépendance de la vitesse de fluage à la valeur $(\tau-\tau$ ) provient du concept de comportement viscoplastique des sols.

La nature et le rôle de la durée de vie de la liaison aux points de contact sont plus embrouillés. Bjerrum (1973) déclare que « si la contrainte de cisaillement est petite la durée de vie sera longue, peut-être des cen- taines ou des milliers d'années ; si la contrainte de cisaillement est grande, la durée de vie sera courte, peut-être seulement des minutes ou des jours $n$. Il est remarquable que la durée de vie des liaisons peut différer par neuf ou dix ordres de grandeur. Ainsi plus le niveau de la contrainte est haut, plus la durée de vie des liaisons est courte et plus le fluage est rapide, et vice versa. On peut admettre que la durée de vie L des liaisons et la vitesse de fluage $\dot{\gamma}$ sont reliées par une relation inverse de proportionnalité et par conséquent on peut écrire :

$$
X=K \frac{F}{R} \frac{\tau-\tau_{p}}{\zeta L}
$$

où $\mathrm{K}$ est un coefficient de proportionnalité ayant la dimension d'un temps et $\zeta$ est la viscosité statique du sol avec la dimension d'une contrainte [ML $\left.{ }^{-1} \mathrm{~T}^{-2}\right]$.

Il est naturel de suggérer que la durée de vie moyenne des liaisons dépend de la direction de l'évolution de la structure du sol et de l'âge de l'application de la contrainte. L'évolution des structures peut conduire soit à une mobilisation des réserves de résistance au cisaillement, soit à une accélération de la déformation, soit à sa stabilisation avec le temps ou soit mené à une rupture totale. Toutes ces possibilités sont réelles, On distingue ainsi quatre phases de fluage correspondantes.

Tous les essais de fluage des sols commencent par la mobilisation des réserves de résistance au cisaillement; c'est la phase de mobilisation. Pendant cette phase il se produit une orientation des particules, le nombre des points de contact entre les surfaces plates des particules en écailles d'argile augmente, la structure du sol devient plus régulière, les forces élémentaires à chaque contact sont plus petites et par conséquent la vitesse de fluage diminue. La vitesse de fluage diminue proportionnellement au temps :

$$
\dot{\gamma}=a \frac{\tau-\tau_{p}}{\zeta} \frac{1}{1}
$$

où a est le coefficient de structure.

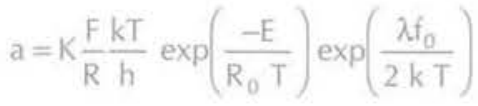

En intégrant l'équation (3), on obtient une équation logarithmique pour la déformation de fluage pour chaque structure dans la phase de mobilisation:

$$
\gamma=\gamma_{0}+a \frac{\tau-\tau_{p}}{\zeta} \ln \frac{t+\Delta t}{\Delta t}
$$

où $\Delta$ t est un petit intervalle de temps égal à une unité, par exemple une seconde, introduit pour une meilleure interprétation mathématique et $\gamma_{0}$ est l'intersection de laxe des $\gamma$ et de la ligne logarithmique.

La phase de mobilisation se développe pendant plusieurs dizaines de jours. La déformation correspondant au minimum de vitesse de fluage est appelée la limite de mobilisation $\mathrm{M}$ et le temps écoulé depuis l'application de la contrainte de cisaillement est appelé le temps de mobilisation $\mathrm{t}_{\mathrm{m}}$.

Après la limite de mobilisation, le fluage au niveau des particules continue différemment en dépendance de la valeur des contraintes de cisaillement. 
Sous des conditions de cisaillement bas, le processus de mobilisation de la résistance au cisaillement se transforme progressivement en stabilisation, ce qui se produit au cours d'une longue période de temps. Ainsi pour des contraintes de cisaillement basses, il existe seulement une phase de mobilisation-stabilisation. Les équations (3) et (4) pour la vitesse de fluage et pour la déformation de fluage sont toujours valables.

Sous des contraintes de cisaillement moyennes après la limite de mobilisation, la désorientation des particules en écailles commence, la durée de vie moyenne des liaisons décroît, et une accélération du fluage se produit. Pendant la phase d'accélération la vitesse de fluage est :

$$
\dot{\gamma}=a \frac{\tau-\tau_{p}}{\zeta t_{m}^{2}} t
$$

En intégrant cette équation, on obtient une équation quadratique pour la déformation de fluage dans la phase d'accélération :

$$
\gamma=\gamma_{0}+a \frac{\tau-\tau_{p}}{2 \zeta t_{m}^{2}} t^{2}
$$

où $\gamma_{0}$ est l'intersection de l'axe des $\gamma$ par la parabole.

Cependant ce n'est pas tout. La vitesse de fluage augmente jusqu'à un maximum et après cela elle décroît de nouveau. La déformation correspondant au maximum de la vitesse de fluage est appelée la limite de stabilisation $S$, et le temps passé le temps de stabilisation $t_{\text {s. }}$ Cet intervalle de temps est assez long, de l'ordre de quelques centaines de jours. Après la limite de stabilisation le fluage décéléré reprend et il peut continuer à se produire pendant des années. Cette phase est appelée la phase de stabilisation. Comme cette phase est physiquement en analogie avec la phase de mobilisation elle est décrite par les mêmes équations (3) et (5).

Sous des contraintes de cisaillement hautes, après la limite de mobilisation, la rupture des structures du sol et l'accélération du fluage commencent et se terminent par la rupture totale du sol. Ce mécanisme se distingue de l'accélération observée sous des contraintes de cisaillement moyennes ; des changements plus profonds se produisent dans la structure du sol. Ceci est appelé la phase de rupture. La vitesse de fluage dans la phase de rupture pour chaque structure est uniforme et égale à :

$$
\dot{\gamma}=a \frac{\tau-\tau_{p}}{\zeta} \frac{t_{1}}{t_{m}^{2}}
$$

où $t_{1}$ est le temps de formation d'une structure donnée.

En intègrant cette équation, on obtient l'équation linéaire de la déformation de fluage pendant la phase de rupture :

$$
\gamma=\gamma_{0}+a \frac{\tau-\tau_{p}}{\zeta} \frac{t_{1}}{t_{m}^{2}} t
$$

où $\gamma_{0}$ est l'intersection de l'axe des $\gamma$ avec la ligne droite représentative.

La vitesse de fluage aux niveaux moléculaire et des particules est déterministe.

\section{3}

\section{Le niveau structural de la déformation du sol}

C'est une caractéristique des sols que la déformation de fluage et la vitesse de fluage sont liées par une relation définie. La nature de cette dépendance n'est pas complètement comprise pour le moment, mais son existence a été prouvée par tous nos essais sans exception: elle est définie par des tendances de développement conditionnées par la causalité. Cette relation est exprimée par les courbes intrinsèques.

Ainsi une contradiction apparait puisque le fluage, pour chaque structure de sol ne peut seulement se produire qu'en suivant les équations (3-9) et donc le déroulement intrinsèque nécessaire du phénomène ne peut être assuré depuis le début jusqu'à la fin. Cette contradiction est résolue de la façon suivante. Le phénomène de fluage pour chaque structure de sol se produit en accord avec ces équations mais quand l'écart par rapport au déroulement nécessaire dépasse une certaine quantité, la structure du sol devient inadéquate. Alors une réorganisation de la structure comme par un saut (RSS) se produit, et le phénomène reprend son propre cours en accord avec le principe de Le Chatelier qui s'énonce : le système tend vers des changements tels qu'ils minimisent l'action des forces extérieures.

Lorsque cela se produit le réarrangement structural des particules de sol et des points de contacts entre elles change brusquement, l'amplitude et la direction des forces élémentaires qui agissent aux points de contact sont modifiées et en conséquence la déformabilité du sol change aussi. Toutes ces quantités changent au hasard. Une nouvelle structure de sol est formée, la vitesse de fluage fait un saut et le fluage continue aux niveaux moléculaire et des particules avec une nouvelle valeur du coefficient a. Alors le RSS n'est plus un phénomène arbitraire hors de propos mais un moyen de régulation des mécanismes de fluage.

La déformation du sol au niveau structural est un processus stochastique.

\section{La déformation du sol au niveau des agrégats}

Les valeurs probabilistiques des caractéristiques mécaniques des structures de sol qui se produisent comme le résultat des RSS ne doivent pas être interprétées comme s'il existait un chaos d'accidents incontrôlés et que les régularités engendrées par le rapport de causalité étaient niées. Elles doivent être considérées comme des causalités montrant des tendances au développement et non comme le signe d'une clarté précise et sans ambiguité des résultats. La probabilité d'atteindre des quantités fortuites doit plutôt être comprise comme une certaine érosion de leur valeur décrite par des intervalles plus ou moins étroits.

\section{3}

\section{Quatre domaines de valeurs des niveaux de contrainte de cisaillement}

Le comportement rhéologique des sols dépend à la fois des contraintes de cisaillement et des contraintes normales. Pour la clarté de la présentation on va exa- 
miner d'abord la dépendance de la déformation de fluage des contraintes de cisaillement en supposant les contraintes normales constantes. L'influence des contraintes normales sera prise en considération ultérieurement.

Quatre domaines de niveau de contrainte de cisaillement peuvent être distingués en dépendance des comportements qualitativement différents des sols (fig. 3).

\section{4}

\section{Le domaine des très basses contraintes de cisaillement}

Les très basses contraintes de cisaillement $(0<\tau<\tau)$ sont trop petites pour produire des déformations à long terme de cisaillement. $\tau_{0}$ est appelée la limite de rigidité. Terzaghi (1931) l'a appelée « point d'écoulement » ou " résistance de liaison $n$; il a indiqué que sous l'influence d'une contrainte d'intensité croissante le point d'écoulement est obtenu dès que la contrainte atteint la résistance des liaisons engendrées par la partie solide du film d'eau absorbée.

Le graphique de la déformation de cisaillement en

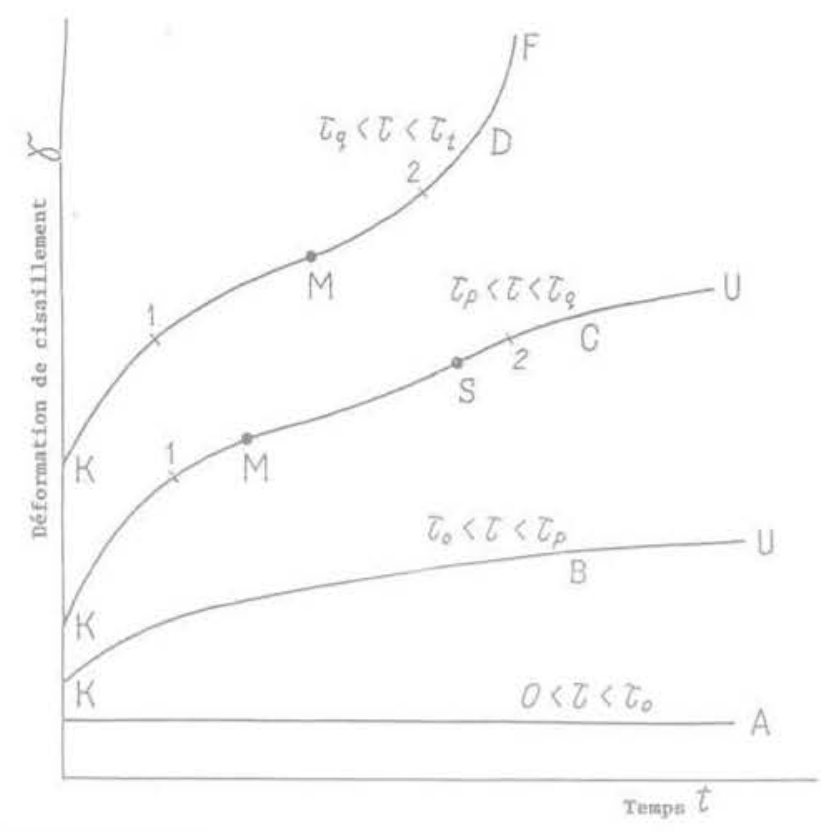

FIG 3 Interprétation proposée des courbes de fluage.

A - Absence de fluage sous des contraintes de cisaillement très basses. B - Une seule phase de fluage séculaire sous contraintes de cisaillement basses (KU phase de mobilisation-stabilisation). $\mathrm{C}$ - Fluage à trois phases sous contraintes de cisaillement moyennes (KM phase de mobilisation, MS phase d'accélération et SU phase de stabilisation).

D - Fluage à deux phases sous contraintes de cisaillement hautes (KM phase de mobilisation et MF phase de rupture). Proposed interpretation of creep curves : A - absence of creep at very low shear stresses; $\mathrm{B}$ - one phase of secular creep at low shear stresses (mobilization-stabilization phase KU) ; $\mathrm{C}$ - three phases of creep at medium shear stresses (mobilization phase KM, acceleration phase MS and stabilization phase SU):

D - two phases of creep at high shear stresses (mobilization phase KM and rupture phase MF). fonction du temps est une ligne droite A parallèle à l'axe des abscisses (Fig. 3). Comme dans les autres cas l'intersection de la courbe de fluage avec l'axe des ordonnées correspond à la déformation (élastique et inélastique) instantanée de cisaillement.

\section{$4,3,2$}

\section{Le domaine des basses contraintes de cisaillement}

Les basses contraintes de cisaillement $\left(\tau_{0}<\tau<\tau_{0}\right)$ sont capables de produire une légère déformation de cisaillement ralentissant avec le temps et détectable seulement par des mesures très précises ou par des observations à très long terme; $\tau$ est appelée la limite de fluage. Pour des contraintes de cisaillement inférieures à cette limite le fluage a un caractère séculaire. Terzaghi (1931) a proposé le terme « résistance au cisaillement ») car c'est la contrainte minimale pour provoquer une déformation de cisaillement active. Le mécanisme de fluage consiste en la seule phase de mobilisation-stabilisation : d'abord la résistance au cisaillement est mobilisée et ensuite la déformation de cisaillement est stabilisée. La convexité de la courbe de fluage B est tournée vers le haut (Fig. 3).

\section{3is \\ Le domaine des contraintes de cisaillement moyennes}

Les contraintes de cisaillement moyennes $\left(\tau_{\mathrm{p}}<\tau<\tau_{\mathrm{q}}\right)$ sont capables d'engendrer un fluage notable; $\tau_{c}$ est appelée la limite de rupture. Le phénomène de fluage comporte alors trois phases :

(a) la phase de mobilisation, quand la vitesse de déformation décroît progressivement et atteint un minimum à la limite de mobilisation $\mathrm{M}$;

(b) la phase d'accélération, quand la vitesse de fluage croît et atteint un maximum à la limite de stabilisation S ;

(c) la phase de stabilisation, quand la vitesse de fluage décroît à nouveau et la déformation après un long terme s'approche de sa valeur finale correspondant à la stabilisation totale du sol.

La décroissance de la vitesse de fluage après sa croissance au cours de la phase d'accélération a été observée pour la première fois par Bishop et Lovenbury (1969); ils ont appelé ce phénomène l'instabilité limitée.

La courbe de fluage $C$ est composée de trois morceaux dont les convexités sont tournées alternativement vers le haut, puis vers le bas et enfin à nouveau vers le haut (Fig. 3).

\section{4}

\section{Le domaine des hautes contraintes de cisaillement}

Les hautes contraintes de cisaillement $\left(\tau_{\mathrm{g}}<\tau<\tau\right)$ sont capables de provoquer la rupture du sol; $\tau$, est appelé la résistance au cisaillement à long terme du sol. Le phénomène de fluage comporte deux phases :

(a) la phase de mobilisation quand la vitesse de déformation de cisaillement décroît progressivement et atteint un minimum à la limite de mobilisation $\mathrm{M}$;

(b) la phase de rupture quand la vitesse de fluage croît sans cesse et mène le phénomène jusqu'à la rupture totale F. 


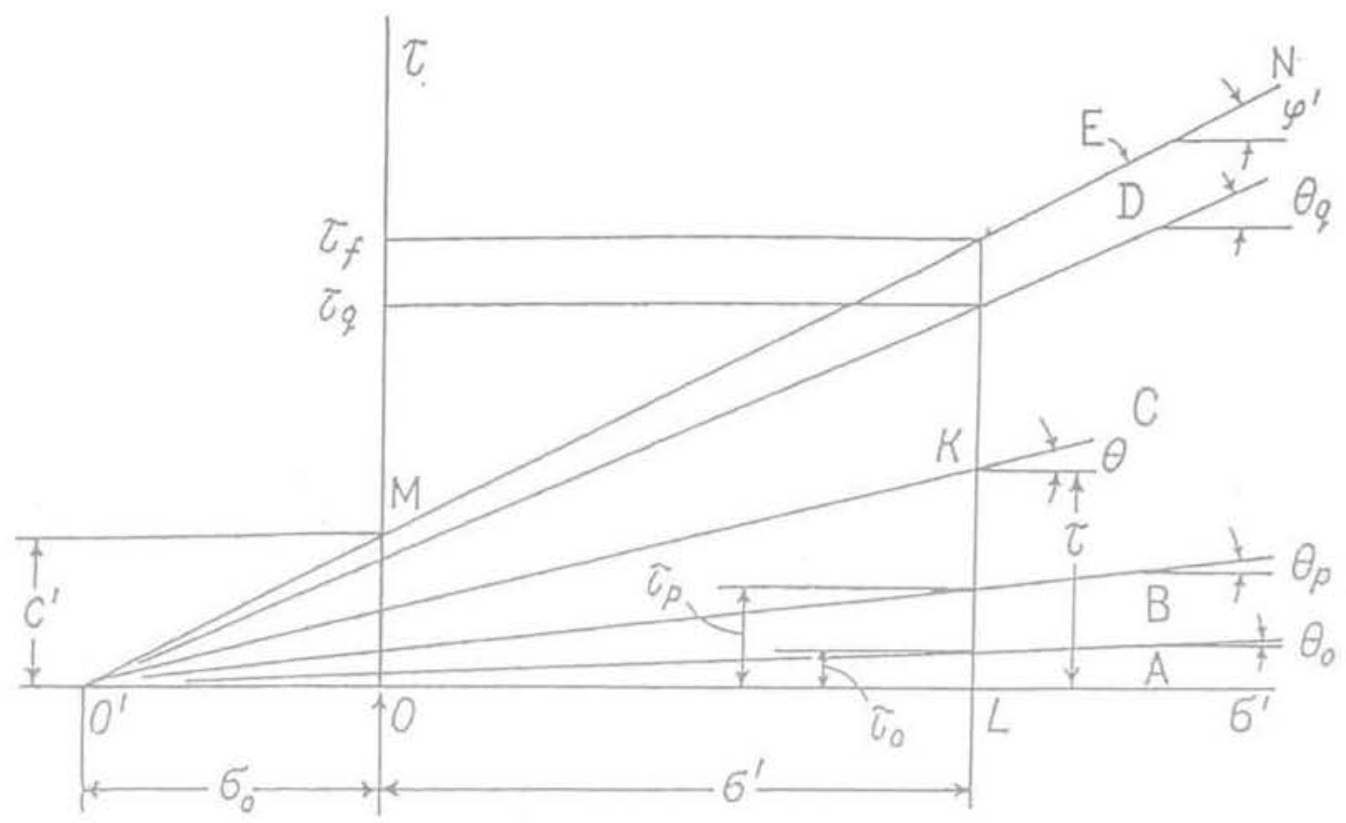

flo. Coefficient de résistance au cisaillement mobilisée.

MN - relation entre la contrainte normale effective $\sigma^{\prime}$ et la résistance au cisaillement maximale (valeur de pic) $\tau_{f}$ (ligne Coulomb-Terzaghi) ;

$c^{\prime}$ - cohésion effective et $\varphi^{\prime}$ angle de frottement interne effectif; $\sigma_{0}$ pression équivalente à la cohérence effective ; $\tau_{0}$ est la limite de rigidité ; $\tau_{p}$ est la limite de fluage ; $\tau_{q}$ est la limite de rupture; $\tau_{p}$ est la résistance maximale du sol.

Domaines de déformation : A - domaine de rigidité (absence de fluage),

B - domaine de fluage séculaire,

C - domaine de fluage décéléré,

$\mathrm{D}$ - domaine de fluage accéléré.

Coefficient of mobilized shear strength.

$\mathrm{MN}$ - relation between the normal effective stress $\sigma$ and peak value of the shear strength $\tau_{\text {, }}$ (Coulomb-Terzaghi line) ; $c^{\prime}$ - effective cohesion and $\varphi^{\prime}$ effective angle of internal friction ;

$\sigma_{0}$ - all-round pressure equivalent to effective cohesion, $\tau$ is the rigidity limit, $\tau$ is the creep limit, $\tau$ is the rupture limit, $\tau_{r}$ is the peak strength of soil. Ranges of deformation: A - range of rigitidy (absence of creep), B - range of secular creep, C - range of decelerating creep.

D - range of accelerating creep.

La courbe de fluage D comporte deux parties dont la convexité est dirigée d'abord vers le haut, ensuite vers le bas.

On remarquera que si on prend pas un soin suffisant pour analyser avec précision la courbe de fluage, les sections entre les points 1 et 2 des courbes expérimentales C et D (Fig. 3) peuvent être considérées comme des segments de droites et on pourrait croire ainsi que l'existence d'un fluage secondaire ayant lieu à vitesse constante a été démontrée expérimentalement.

\section{Coefficient de résistance au cisaillement mobilisée}

L'application directe des critères qui viennent d'ètre décrits aux talus ou aux fondations est difficile parce que l'état de contraintes des massifs de sol est caractérisé par une grande différence des valeurs absolues des composantes normales et tangentielles des contraintes aux divers points du volume. II est opportun toutefois d'utiliser au lieu de contraintes de cisaillement le concept moins variable de coefficient de résistance au cisaillement mobilisée (Fig. 4).

$$
\operatorname{tg} \theta=\frac{\tau}{\sigma_{0}+\sigma^{\prime}}
$$

où $\sigma_{0}=c^{\prime} \cot \varphi^{\prime}$ est une pression fictive équivalente à la cohésion effective, $c^{\prime}$ et $\varphi^{\prime}$ les paramètres de la résistance en cisaillement en terme de contraintes effectives $\tau_{f}=c^{\prime}+\sigma^{\prime} \operatorname{tg} \varphi^{\prime}$ et $\tau_{\text {, est la résistance maximale du sol }}$ (résistance de pic).

Le coefficient de résistance au cisaillement mobilisée $\operatorname{tg} \theta$ est la tangente de l'angle d'obliquité, ou angle formé par la résultante des contraintes effectives totales, les contraintes internes inclues, avec le plan sur lequel ces contraintes agissent. De la même façon, la frontière entre les zones de fluage décéléré et accéléré est déterminée par la valeur du coefficient de résistance au cisaillement mobilisée à la rupture :

$$
\operatorname{tg} \theta_{q}=\tau_{q} /\left(\sigma_{0}+\sigma^{\prime}\right)
$$

\section{2}

\section{Faits expérimentaux}

Un ensemble d'essais drainés de fluage a été effectué avec un appareil de cisaillement annulaire sur des échantillons intacts d'une argile pliocène lacustre à diatomées surconsolidée, prélevée dans le tunnel de déviation du réservoir d'eau de Shamb à Sissian, Arménie, à des profondeurs de $z=40$ et $76 \mathrm{~m}$. La durée de quelques essais a été de plus de 2300 jours, soit plus de six ans. Leurs caractéristiques sont indiquées dans le tableau. Vingt essais ont été menés en tout ( $\mathrm{N}^{\circ}$ 12-31). 


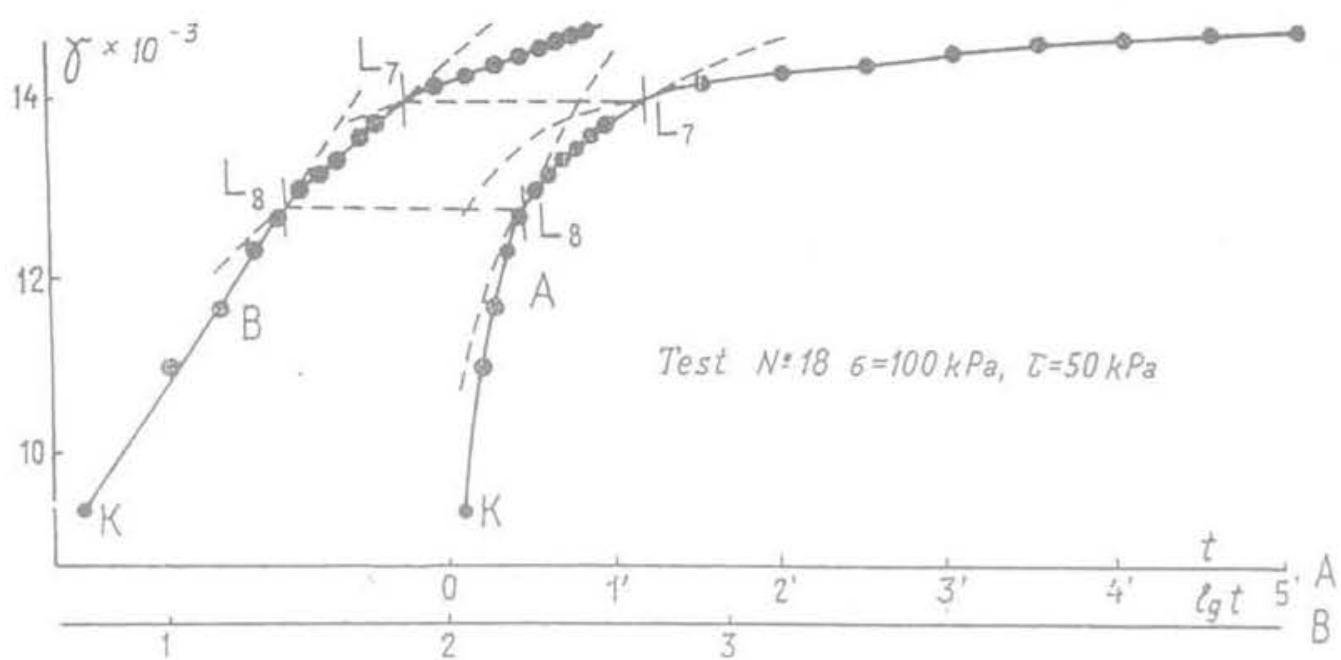

FG.S Linéarisation des points expérimentaux dans le graphique semi-logarithmique B pour un essaí effectué sous des hautes contraintes de cisaillement pendant les cinq premières minutes. A est le graphique en coordonnées arithmétiques.

Linearization of experimental points in the semilogarithmic graph B of a test run at high shear stresses for the first 5 minutes. $A$ is the arithmetical graph.

Tous les essais ont été considérés dans cette analyse, et aucun point expérimental n'a été omis.

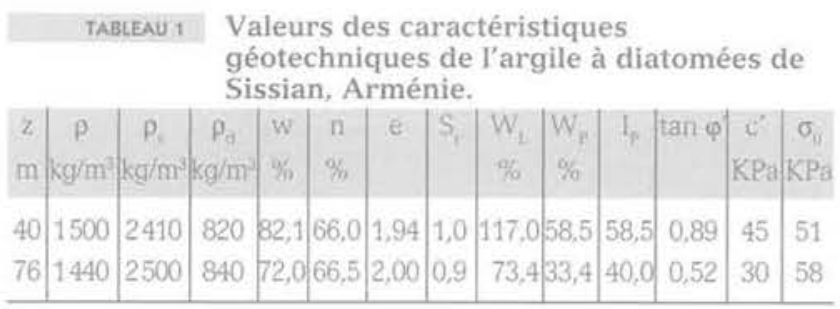

Les essais ont été effectués dans le laboratoire de géomécanique de l'Institut de Géologie de l'Académie des sciences d'Arménie à Erevan. Les essais ont été réalisés avec une précision extrême par Mme l'Ingénieur en chef Paitzar Mazedjan, actuellement à Paris.

\section{4}

\section{Linéarisation des points expérimentaux}

La linéarisation des points sur les graphiques avec des échelles fonctionnelles est une méthode aisée et convaincante pour déterminer les équations décrivant une collection donnée de points. La linéarisation des points appartenant à la même structure de sol sur un diagramme semi-logarithmique est la preuve la plus évidente et la plus claire que le phénomène de fluage pour chaque structure peut être représenté par une équation logarithmique ; d'une façon analogue la linéarisation des points sur un diagramme semi-quadratique ou arithmétique confirme la validité d'équations quadratiques ou linéaires. La linéarisation des points expérimentaux dans différents diagrammes est montrée dans les figures suivantes.

Les résultats de la première étape d'un essai effectué sous de hautes contraintes de cisaillement pendant les cinq premières minutes est représenté sur la fig. 5. La ligne A est la relation entre le temps et la déformation de cisaillement en échelle arithmétique; elle apparaît comme une courbe régulière. Si on la reporte sur le diagramme semi-logarithmique B, la ligne A apparait comme l'intersection de trois segments de droite ce qui montre que la ligne A est composée en réalité des morceaux de trois courbes logarithmiques. Les parties non utilisées de ces courbes sont indiquées en pointillés.

La figure 6 montre les résultats d'un essai effectué sous de hautes contraintes de cisaillement en échelle arithmétiques, la ligne A pour les trente premières minutes, la ligne B pour les cinq premières heures. Ces deux courbes sont à peu près lisses. Toutefois après avoir reporté les résultats des essais en échelle semilogarithmique $\mathrm{C}$ quatre lignes droites clairement définies peuvent être distinguées pour les structures de sol successives.

La figure 7 montre les résultats d'un essai effectué sous des contraintes de cisaillement moyennes pendant les cinquante-deux premiers jours. Le graphique montre le domaine au voisinage de la limite de mobilisation $\mathrm{M}$. Le graphique $\mathrm{A}$ est dessiné avec une échelle arithmétique, le graphique B est semi-logarithmique et le graphique $C$ est semi-quadratique. Les graphiques $B$ et $C$ confirment ce qui a été dit plus haut relativement aux changements des structures de sol.

La même collection de points expérimentaux a été présentée sur la figure 2 comme exemple de la dispersion des points expérimentaux, inexplicable par des erreurs de mesure. Il est évident d'après la figure 6 qu'après la séparation des structures de sol et la détermination de la limite de mobilisation $\mathrm{M}$, la dispersion des points acquière une signification profonde. Cela démontre le caractère régulier de la déformation des structures de sol au cours de la durée de vie de chaque structure, alternant avec les réorganisations de cette structure par sauts (RSS).

Par le résultat de la linéarisation des points expérimentaux des courbes de fluage dégénèrent en lignes brisées dans des graphiques semi-logarithmiques et semi-quadratiques. La courbe dans le système de coordonnées $(t, \gamma)$ est constituée par des morceaux de courbes logarithmiques ou de paraboles pour les structures successives.

Deux modes de linéarisation en échelle arithmétique ont été observés : une croissance régulière de la 




FG.6 Linéarisation des points expérimentaux dans le diagramme semi-logarithmique C d'un essai effectué sous hautes contraintes de cisaillement pendant les cinq premières heures. A et B sont des graphiques en coordonnées arithmétiques.

Linearization of experimental points in the semilogarithmic graph $C$ of a test run at high shear stresses for the first 5 hours. A and B are the arithmetical graphs. vitesse de fluage pour les structures de sol successives (Fig, 8 et 11) et des oscillations de relaxation à longue période (Fig. 9). Ces modes de linéarisation peuvent être interchangés.

La transition angulaire d'une ligne droite vers l'autre dans tous ces cas démontre tout à fait distinctement que les réorganisations des structures du sol dans les moments de la RSS passe d'une courbe possible, logarithmique, parabolique ou ligne droite - à la suivante en la recoupant.

\section{2}

\section{Courbes complètes de fluage}

Les résultats des essais de fluage sont présentés complètement en deux diagrammes sur les figures 10 et 11. Pour plus de clarté quelques points expérimentaux situés exactement sur les lignes n'ont pas été tracés sur ces graphiques.

Les résultats d'un essai effectué sous des contraintes de cisaillement moyennes sont montrés sur la figure 10. Les échelles et les courbes correspondantes sur les diagrammes sont notées par les lettres $\mathrm{A}$ à $\mathrm{P}$. Le diagramme montre que 7 RSS (de L à L.) se sont produits au cours de la phase de mobilisation depuis le début $\mathrm{K}$ des observations jusqu'à la limite de mobilisation M (de cinq secondes à quarante-deux jours), 6 RSS (de $Q_{1}$ à $Q_{6}$ ) dans la phase d'accélération jusqu'à la limite de stabilisation S (217 jours) et 8 RSS (de $R_{1}$ à $R_{8}$ ) dans la phase de stabilisation jusqu'à la fin des observations U (952 jours) et encore 2 RSS à la limite de mobilisation $\mathrm{M}$ et à la limite de stabilisation $\mathrm{S}$.

Les résultats d'un essai effectué sous hautes contraintes de cisaillement sont montrés sur la figure 11. Le phénomène de fluage s'est terminé par la rupture totale. La linéarisation des points expérimentaux dans la phase de rupture est représentée sur un graphique arithmétique.

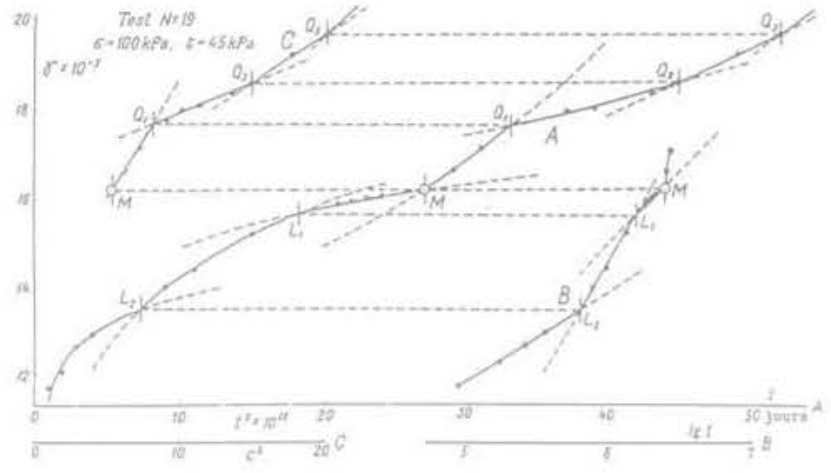

FIG.7 Linéarisation des points expérimentaux dans le diagramme semi-logarithmique B avant la limite de mobilisation $\mathrm{M}$ et dans le diagramme semi-quadratique $\mathrm{C}$ après cette limite pour l'intervalle de temps entre 1 et 52 jours. A est le diagramme en coordonnées arithmétiques.

Linearization of experimental points in semilogarithmic graph B before the mobilization limit $M$ and in semiquadratic graph $\mathrm{C}$ after this limit for the time interval from 1 to 52 days. $A$ is the arithmetical graph.



FG. L Linéarisation des points expérimentaux en coordonnées arithmétiques dans la phase de rupture sous hautes contraintes de cisaillement. Augmentation régulière de la vitesse de fluage pour les structures de sol successives.

Linearization of experimental points in the arithmetical graph in the rupture phase at high shear stresses. Steady increase of creep rate for successive soil structures. 


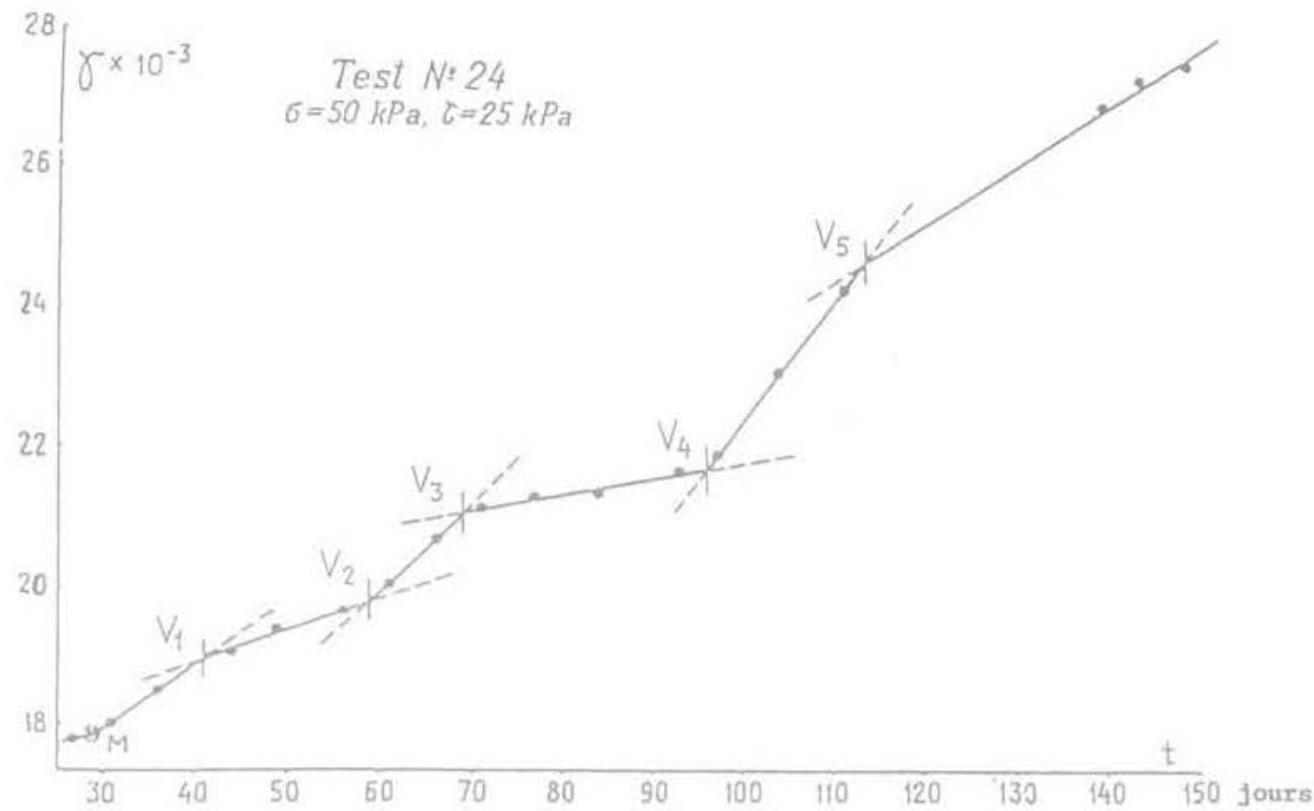

FIG.9 Linéarisation des points expérimentaux en coordonnées arithmétiques sous des contraintes de cisaillement égales à la limite de rupture. Oscillations de relaxation à longue période.

Linearization of experimental points in the arithmetical graph at shear stresses equal to the rupture limit. Longperiodical relaxation oscillations.

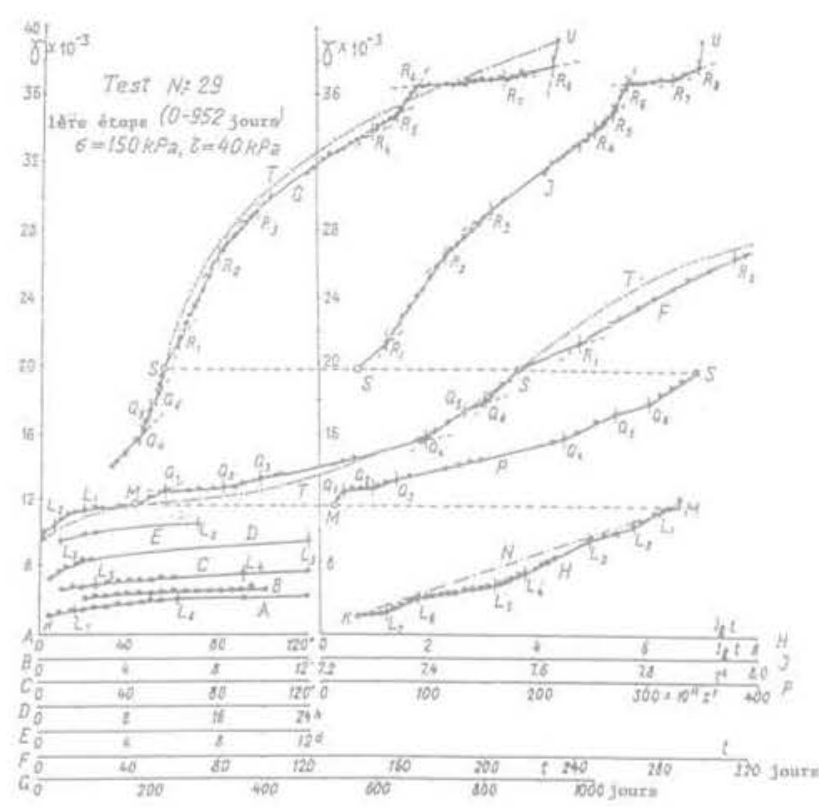

BG.10 Courbes de fluage pour des essais effectués sous des contraintes de cisaillement moyennes. Linéarisation dans les diagrammes semi-logarithmiques $\mathrm{H}$ et $\mathrm{J}$, et dans le diagramme semi-quadratique P. Les symboles A, B.... sur les échelles et sur les courbes montrant les correspondances entre elles. Creep curves of a test run at medium shear stresses. Linearization in the sernilogarithmic graphs $\mathrm{H}$ and $\mathrm{J}$, and in the semiquadratic graph P. Symbols A, B,... at scales and curves show the correspondence between them.

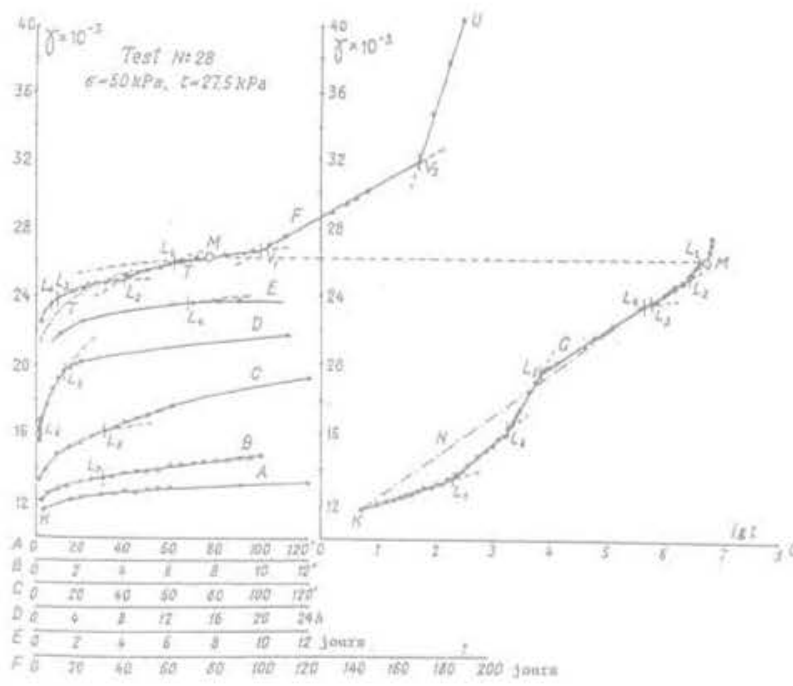

HG.11 Courbes de fluage pour un essai effectué sous des contraintes de cisaillement hautes. Linéarisation dans le diagramme arithmétique $\mathrm{F}$ dans la phase de rupture MU. Augmentation régulière de la vitesse de fluage pour des structures de sol successives. Pour des explications voir la fig. 10 . On voit d'après les courbes A et $\mathrm{E}$ pour différentes échelles de temps, que chacune d'elles se termine presque par une portion de droite et ainsi la transition des courbes vers la vitesse de fluage constante est seulement le résultat d'un effet d'échelle.

Creep curves of a test run at high shear stresses. Linearization in the arithmetical graph F in the rupture phase MU ; steady increase of the creep rate for successive soil structures. For explanations see fig. 10. It is seen from curves A to E for different time scales, that each one of them ends by an almost straight portion, and therefore the transition of curves to the constant creep rate is the result of the scale effect only. 
Il est évident d'après ces deux dernières figures que l'extrapolation des courbes de fluage sur une longue durée est impossible. Aucune extrapolation ne peut donner les courbes $F$ et $G$ pour plusieurs centaines de jours à partir des courbes $E$ déterminées pendant les douze premiers jours.

Que la linéarisation des points expérimentaux après la limite de mobilisation $\mathrm{M}$ se produise en échelle semiquadratique ou en échelle arithmétique est de première importance puisque cela rend possible de déterminer correctement et en son temps dans quel domaine de contraintes de cisaillement (moyennes ou hautes) l'essai est conduit. Ainsi, il n'est pas nécessaire de mener l'essai jusqu'à la rupture complète de ce qui peut nécessiter de nombreux mois ou même d'années. Ce fait facilite aussi la détermination de la limite de rupture $\tau_{\mathrm{q}}$

\section{3}

\section{Énergie dissipée dans les phases d'accélération et de rupture}

Tous les essais de cisaillement commencent par la phase de mobilisation. Les phases d'accélération et de rupture viennent après la limite de mobilisation, respectivement à moyennes et à hautes contraintes de cisaillement. La comparaison et l'analyse du comportement des sols dans ces phases sont d'une grande importance pratique.

La vitesse de fluage pour chaque structure de sol dans la phase d'accélération sous contraintes de cisaillement moyenne croit en fonction du temps alors qu'elle reste constante dans la phase de rupture, sous hautes contraintes de cisaillement. Le fluage accéléré est plus favorable que le fluage à vitesse constante au sens de la consommation d'énergie. L'énergie dissipée dans le dernier cas est plus grande puisqu'elle est associée à des fluctuations qui ne disparaissent pas. La situation est en analogie avec la transition entre l'écoulement laminaire, décrit par la loi de Darcy, qui est plus favorable aux bas gradients hydrauliques, et l'écoulement turbulent moins favorable sous des gradients hydrauliques élevés, avec ses pertes d'énergie à cause de la turbulence et des remous.

On a remarqué que sous des contraintes de cisaillement un peu plus petites que la limite de rupture, les points expérimentaux s'alignent en échelle arithmétique bien que le phénomène se termine par la stabilisation. Ceci montre le fait que la transition entre la phase de mobilisation et la rupture commence par une augmentation des fluctuations et de l'énergie dissipée.

Les comportements différents des sols dans les phases d'accélération et de rupture sont probablement dus à la distinction entre des formes d'auto-organisation de la substance du sol sous des contraintes de cisaillement moyennes ou hautes. Les forces de dissipation jouent un rôle important dans ces phénomènes. Les pertes d'énergie les plus petites des mécanismes de fluage ordonné, sous des contraintes de cisaillement moyennes deviennent les pertes d'énergie les plus grandes du fluage désordionné, sous des contraintes de cisaillement hautes. En conséquence les fluctuations sous des contraintes de cisaillement hautes augmentent considérablement, le système devient instable et le mécanisme se transforme en une autre forme d'autoorganisation de la matière (Haken, 1960).

\section{Courbes intrinsèques}

La relation entre la déformation de cisaillement et la vitesse de déformation de cisaillement au cours de l'ensemble du phénomène de fluage est une caractéristique importante des rapports de cause à effet qui commandent la déformation du sol.

Pour tenir compte de la longue durée des essais de grande qualité et de l'importance des processus qui se produisent dans l'étape initiale de l'essai cette relation est présentée en échelle logarithmique : le logarithme de la déformation de fluage en abscisses et le logarithme de la vitesse de fluage en ordonnées. Ce graphique est appelé la courbe intrinsèque.

La courbe intrinsèque pour les essais effectués sous contraintes de cisaillement moyennes et hautes est présentée correspondemment sur les fig. 12 et 13. D'après ces courbes il est manifeste que le cours du fluage prescrit par les équations (3), (5) et (6), montré sur les diagrammes par des lignes fines, diffère de la courbe intrinsèque (tracée en trait fort) et que les RSS ren-

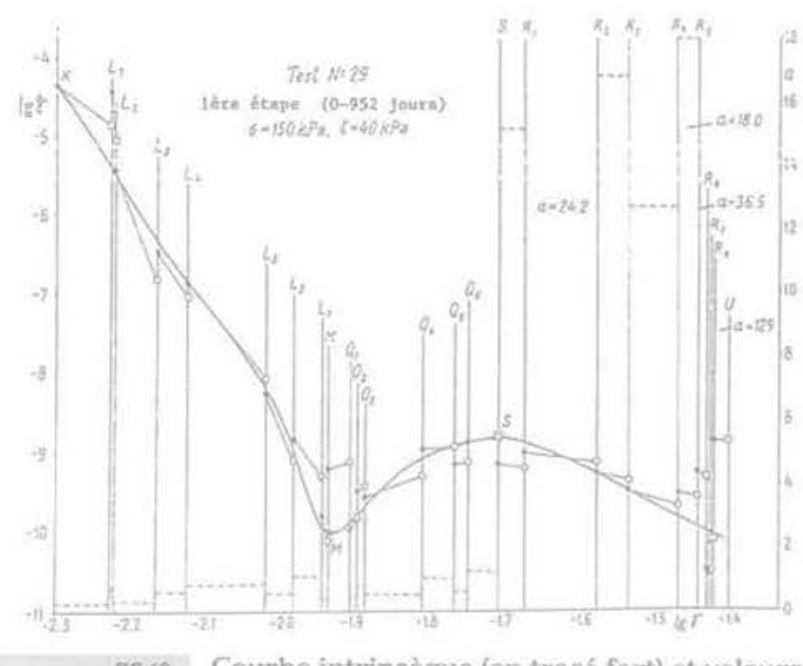

ศG.18 Courbe intrinsèque (en tracé fort) et valeurs du coefficient de structure (lignes tiretées horizontales) d'un essai effectué sous contraintes de cisaillement moyennes. En abscisses sont les logarithmes de la déformation de cisaillement (log $\gamma$ ), en ordonnées, sont à gauche, les logarithmes de la vitesse de fluage (log $\gamma)$ et, à droite, les coefficients de structures a. Les lignes verticales fines représentent les déformations de fluage au moment des RSS. Les cercles blancs représentent les vitesses de déformation avant la RSS, même chose pour les cercles noirs après la RSS. Les lignes fines inclinées montrent le changement de la vitesse de fluage au cours de la durée de vie des structures, Intrinsic curve (heavy line) and structural coefficient values (horizontal dash lines) of a test run at medium shear stresses. Abscissas are logarithms of shear strain (log $\gamma)$, ordinates on the left are logarithms of creep rate (log $\dot{\gamma}$ ) and on the right-structural coefficients a. Vertical thin lines are creep deformations in moments of the Jump-like Structure Reorganisation (JSR). White circles are creep rates before the JSR, black circles ditto after JSR. Inclined thin lines show the change of the creep rate during lifetime of structures. 




voient le phénomène de la position atteinte (cercles blancs) à la ligne intrinsèque. Mais ce retour n'est pas précis, et la nouvelle position après le saut (cercles noirs) n'est pas toujours sur la ligne intrinsèque. Ainsi une certaine irrégularité se produit et les structures de sol nouvellement formées doivent supporter aussi bien les vitesses de fluage les plus grandes ou les plus petites ce qui est nécessaire pour une adéquation totale à la coưrbe intrinsèque.

Néanmoins les points noirs sont situés dans la direction vers la ligne intrinsèque ce qui contredit apparemment la nature stochastique du RSS. En réalité il n'y a pas contradiction mais sélection des structures qui sont produites. Les structures de sol nouvellement formées peuvent avoir différentes valeurs de la déformabilité $F / R$ et des forces élémentaires $\mathrm{f}_{0}$ et par conséquent elles peuvent être plus ou moins adéquates comparées aux structures existantes précédentes qui se sont effondrées. Quoi qu'il en soit, les structures les moins adaptées sont détruites immédiatement et initient la formation de nouvelles, cependant que les plus adaptées sont préservées pendant un certain temps. Ainsi la sélection des structures les plus ajustées se produit, et les mécanismes peuvent satisfaire simultanément les équations (3), (5) et (6) et les propriétés intrinsèques du sol.

\section{5}

\section{Critère de rupture}

D'après les concepts traditionnels la contrainte de cisaillement admissible est déterminée par la valeur de la contrainte de cisaillement telle qu'une augmentation de la vitesse de fluage ne se produise pas, en d'autre terme par la limite de fluage $\tau$. Pour les sols étudiés prélevés à une profondeur de $40 \mathrm{~m}$ et essayés sous une contrainte normale $\sigma^{\prime}=100 \mathrm{kPa}$ on a obtenu $\tau_{\mathrm{p}}=16 \mathrm{kPa}$. Toutefois sous des contraintes de cisaillement moyennes la phase d'accélération passe à la phase de stabilisation et en conséquence la limite supérieure de l'indestructibilité sera la limite de rupture $\tau_{\mathrm{p}}$ qui est égale à $48 \mathrm{kPa}$.

Le rapport entre les limites de rupture et de fluage dans ce cas est égal à trois. Par conséquent, il est possible d'augmenter considérablement la contrainte de cisaillement admissible si des expériences correspondantes étaient faites et que le concept bien fondé de résistance à long terme des sols était utilisé. Naturellement, tout le domaine de la résistance au cisaillement jusqu'à la limite de rupture ne serait pas considéré comme admissible et un certain coefficient de sécurité devrait ètre introduit. Mais néanmoins c'est une immense réserve d'utilisation de la résistance au cisaillement qui reste soit oisive, soit qui est utilisée sans une claire compréhension des mécanismes réels dans les sols, sans avoir la possibilité de mettre en œuvre une approche analytique et avec le risque que le mécanisme de fluage se termine par une rupture dans un avenir lointain.

\section{3}

\section{Concentration du fluage avec le temps}

L'isoligne correspondant au coefficient de résistance au cisaillement mobilisée à la limite de rupture tg $\theta_{0}$ divise le domaine contraint en deux parties qui se dèveloppent dans des directions différentes : la partie externe de fluage décéléré qui va vers la stabilisation lorsque le temps s'écoule, et la partie interne de fluage accéléré qui va jusqu'à la rupture totale. Il en résulte que les larges zones caractéristiques du fluage de la phase initiale du processus vont se rétrécir graduellement et se transformer en de fines surfaces de glissement. Ce phénomène est appelé la concentration du fluage avec le temps.

Le concept de concentration du fluage avec le temps est d'une grande importance pour la compréhension du mécanisme des glissements de terrain et des ruptures des fondations.

Dans la phase préparatoire du glissement de terrain, appelée le fluage profond des pentes, la distorsion se produit dans une large zone. A cause de la concentration du fluage avec le temps des surfaces de glissement se forment dans les zones de fluage accéléré et le processus se termine par un glissement de terrain dans le plein sens du terme. De méme la distorsion à long terme se produit sous les fondations dans les zones de fluage accéléré, des surfaces de glissement se produisent dans le sol et à cause du phénomène de concentration du fluage avec le temps le processus se termine par la rupture de la fondation.

Il en résulte que la rupture d'une pente ou d'une fondation n'a pas le besoin indispensable qu'une nouvelle cause externe soit initiée, comme une forte pluie ou un chargement excessif ; la rupture peut se produire après de nombreuses années simplement comme le résultat de la concentration du fluage avec le temps et du développement intense des déformations de cisaillement.

Cette circonstance peut probablement expliquer quelques cas de rupture qui n'étaient pas adéquats aux 
facteurs qu'on leur attribuait et ainsi restaient énigmatiques.

Les principes de la mécanique des sols basés sur le concept des milieux quasi solides ont été présentés comme un corps de doctrine complet. Le concept des sols considérés comme un milieu dispersé n'exclut pas ces principes. Le sol a une nature duale, il peut être représenté aussi bien par un corps quasi solide ou par un milieu dispersé. Les deux sont simplement des aspects complémentaires de la même réalité.

Le concept des sols considérés comme des corps quasi solides peut être utilisé dans les problèmes de stabilité des pentes et pour la résistance des fondations. Le principe de la rupture progressive des argiles due à la chute de résistance de la valeur maximale (résistance de pic) à la valeur résiduelle est caractéristique pour ce cas, particulièrement lorsqu'il s'agit d'argiles surconsolidées.

Le concept des sols considérés cornme des milieux dispersés peut être utilisé dans les problèmes de déformabilité des sols et des pentes. Le principe de la rupture progressive des argiles due à la concentration du fluage avec le temps est caractéristique de ce cas surtout lorsque les argiles contiennent une forte proportion de montmorillonite.

4

\section{Conclusion}

Les mécanismes de la RSS en cours de cisaillement ont été établis théoriquement et vérifiés expérimentalement. Ces analyses ont conduit à établir un nouveau mécanisme de concentration du fluage avec le temps qui peut être appliqué aussi bien pour les glissements de terrain que pour les fondations.

Le RSS est un phénomène spatial et temporel qui consiste en une auto-organisation de la matière des milieux dispersés (Prigogine, 1980). Elle s'accorcle avec tous les quatre postulats synergiques suivants

(a) le système est ouvert puisqu'il reçoit de l'énergie de l'extérieur aux dépens des forces de cisaillement appliquées ;

(b) le processus est non linéaire puisque la réaction - la RSS - n'est pas proportionnelle à l'action externe;

(c) le phénomène est post-critique puisque la transition par sauts est possible seulement aux points de bifurcation, loin des états d'équilibre thermodynamique, qui sont déterminés par un accord entre la vitesse de fluage réelle pour une valeur donnée de la déformation et la vitesse nécessaire prévue par la courbe intrinsèque; et finalement :

(d) le phénomène est caractérísé par la stabilité de la dissipation spatiale et temporelle, puisque son développement nécessite un échange d'énergie continue avec l'environnement.

La déformation des sols dispersés n'est pas effectivement économique du point de vue de la consommation d'énergie si on la compare avec le développement du phénomène dans les corps solides. La déformation des sols dispersés est accompagnée par de nombreuses réorganisations de la structure, elle prend beaucoup de temps, elle est partiellement indéfinie, elle est caractérisée par des irrégularités de marche et quelquefois des oscillations à long terme se produisent.

L'auto-organisation dispersive des systèmes dissipatifs est capable d'une évolution consistant dans la sélection des structures ajustées les plus adéquates; son comportement est spontané et régulier, l'action de champs organisateurs extérieurs n'est pas nécessaire.

L'analyse des structures du sol et de leur RSS est la clef de la compréhension des phénomènes complexes qui se produisent dans le sol au cours de la déformation. On peut penser que la courbe intrinsèque qui gouverne le cours de la déformation indique la relation optimale entre la déformation de cisaillement et la vitesse de déformation de cisaillement pour que le chemin de déformation soit le plus économique.

La situation peut être comprise comme une version de la triade de Darwin, l'hérédité se manifestant comme le fait que l'état antérieur du sol prédétermine le RSS, la variabilité des mécanismes de saut et finalement la survie des structures de sol les mieux adaptées. La signification pratique du concept proposé consiste dans la possibilité d'apporter une nouvelle approche qualitative pour l'analyse des résultats d'essai de cisaillement et en une augmentation considérable de l'information. Le réseau de déformation impliqué permettant ordinairement de faire seulement des considérations générales et obligeant à rester dans les diagrammes à échelle réduite peut ètre remplacé par une pénétration plus vivante des secrets de la nature.

L'auteur exprime sa gratitude au professeur Pierre Habib pour lui avoir donné l'occasion de présenter cette conférence sur le fluage des sols devant ses collègues de Paris, pour l'avoir assisté pour la traduction française et enfin pour la publication dans la célèbre Revue Française de Géotechnique.

\section{Bibliographie}

Bishop A.W. (1969) - The strength of soils as engineering materials. Sixth Rankine Lecture, Geotechnique, vol. 16, $\mathrm{n}^{\circ} 2$, pp. 91-128.

Bishop A.W. and Lovenbury H.T. (1969) Creep characteristics of two undisturbed clays. Proceedings, Seventh International Conference on Soil Mechanics and Foundation Engineering, Mexico, vol. 1, pp. 29-37.

Goldstein M.N. (1979) - Mechanical properties of soils (Stress-strain and deformation characteristics). Stroiizdat Moscow, 301 p. en russe.

Haken H, (1980) - Advanced Synergetics.
Instabilities hierarchies of self-organizing systems and devices. Berlin: Springer Verlag.

Mitchell J.K. (1964) - Shearing resistance of soils as a rate process. Journal of Soil Mechanics and Foundation Division, American Society of Civil Engineers. vol. 90, SM1, pp. 29-61.

Mitchell J.K. (1993) - Fundamentals of soil behavior. 2nd ed., John Wiley and Sons, New York, $437 \mathrm{p}$.

Prigogine I. (1980) - From being to becoming. Time and complexity in physical sciences. Freeman \& Co, San Francisco.

Schmid W.E, (1962) - New concepts of shearing strength for saturated soils, pt. 2, Soil-Sols, n ${ }^{\circ}$ 2, pp. 19-28.

Ter-Stepanian G. (1975) - Creep of a clay during shear and its rheological model. Geotechnique, vol, 25, $\mathrm{n}^{\circ} 2$, pp. 299-320.

Ter-Stepanian G. (1992) - Mechanics of soil creep during shear. In: Chowdhury R.N. (editor), Geomechanics and Water Engineering in Environmental Management, A.A. Balkema, Rotterdam, pp. 529-557.

Terzaghi Ch. (1931) - The static rigidity of plastic clays. Journal of Rheology, vol. 2 , $n^{\circ} 3$, pp. 253-262. 